\title{
Ethique et violence chez Audrée Wilhelmy
}

\author{
Marie-Hélène Larochelle \\ Université York
}

\begin{abstract}
Résumé
Plusieurs fils pourraient être tirés des textes d'Audrée Wilhelmy. Le roman est un jute rêche et dense, mais fragile : quand on tire un brin c'est tout l'ensemble qui s'effiloche. La sélection est délicate donc. Cet article-entretien propose de suivre le fils du féminisme, dans son rapport avec les théories du Care, sachant qu'il ne sera pas possible d'aller au bout sans créer quelques nœuds.

La fluidité de l'écriture va de pair avec un accueil inconditionnel de la nature. Il n'y a pas ici de sélection; la vague est aussi écume; le bois, fongus; l'animal, carcasse; le suave, puanteur. Chaque élément a sa place, joue un rôle dans cet organisme. L'animalité des personnages est ainsi une forme d'harmonie, un respect de l'instinct qui leur permet souvent de se fondre dans leur habitat. La nature est un accomplissement non pas une régression. En accepter la force, la dureté, l'ambition, est le propre des élus. Les personnages d'Audrée Wilhelmy sont tous des exceptions.

Des conditions de la lecture, c'est la sauvagerie et la vivacité qu'on retient. Que faire de ces femmes soumises qui se laissent battre, violer, tuer? Ces femmes qui ne fuient pas? Ces femmes qui se taisent? C'est cette violence brute du féminin que cet article souhaite comprendre. Le moment où le langage du corps est rendu à l'esthétique.
\end{abstract}

\footnotetext{
Abstract

Several threads could be drawn from the texts of Audrée Wilhelmy. The novel is a rough and dense, but fragile: when you pull a strand, the whole thing unraveled. The selection is therefore delicate. This article-interview proposes to follow the feminism pattern, in its relation to the theories of Care, knowing that it will not be possible to go to the end without creating a few knots.

The fluidity of writing goes with an unconditional reception of nature. Each element has its place, plays a role in this organism. The animality is a form of harmony, a respect for the instinct which allows to blend into habitat. Nature is an accomplishment not a regression. Accepting its strength, toughness and ambition is something that is chosen. Audrée Wilhelmy's characters are all exceptions.

Reading conditions are savagery and liveliness that is retained. What to do with these submissive women who let themselves be beaten, raped, killed? These women who don't run away? These women who are silent? It is this raw violence of the feminine that this article wishes to understand. The moment when body language is restored to aesthetics.
} 
Plusieurs fils pourraient être tirés des textes d'Audrée Wilhelmy. Le roman est un jute rêche et dense, mais fragile : quand on tire un brin c'est tout l'ensemble qui s'effiloche. La sélection est délicate donc. Je propose de suivre le fils du féminisme, dans son rapport avec les théories du Care, sachant qu'il ne sera pas possible d'aller au bout sans créer quelques nœuds.

La métaphore du tissu n'est pas la mienne, c'est celle qui ouvre Le corps des bêtes :

Mie le fait encore. Elle chiffonne son esprit, elle imagine qu'elle tire une ficelle et qu'alors il sort par son oreille, il chatoie devant elle, malléable comme une retaille de tissu, elle le roule serré et le glisse dans un autre cerveau, celui du poisson qui va périr, celui d'une fourmi ou de l'un des très grands cerfs qui brament à l'orée de la forêt. (Wilhelmy, 2017, p. 11)

La poésie de Wilhelmy n'a pas d'âge. Elle est artisanat. Savoir-faire brut. Unique et intemporel à la fois. Et foncièrement féminin. Féministe. Comme le secret d'une grand-mère, comme le savoir-faire ésotérique qu'on transmet de mère en fille, son écriture crée ses propres filiations en resserrant une compétence à laquelle participe le lecteur.

Sans être une suite de Oss, son premier roman, Le corps des bêtes en reprend une héroïne, Noé, femme sauvage et muette, qui a maintenant une fille, Mie. Mie a douze ans. Elle découvre son corps et sa sensualité avec maturité et indépendance. Elle veut que son oncle la prenne comme une femme, comme une bête. 
Entre les deux Les Sangs, récit poétique qui repensent la destinée des femmes de Barbe-Bleue, ici nommé Féléor, est construit d'une autre matière, mais n'est pas incohérent avec l'ensemble, au contraire, puisqu'il approfondit, en d'autres termes, des traits de l'esthétique de l'auteure que confirme le roman suivant.

Ces trois romans ont en commun de se construire autour de femmes fortes, violentes et indomptables. Ce choix en lui-même pourrait selon moi qualifier l'œuvre de féministe. Mais l'analyse est plus complexe; nous le verrons. Suivons néanmoins ce premier fil, celui des femmes sauvages, pour voir jusqu'où il peut nous mener avant de se rompre.

Dans Oss, Noé était une enfant trouvée que les villageois de Oss violentaient sans que la détermination et l'entêtement de l'enfant ne soient abimés : «Elle se retourne, il n’y a rien de naïf dans ses yeux ; elle est grande, longue, mais elle n'est pas fragile » (Wilhelmy, 2011, p. 61). Noé se nourrissait de la peur qu'elle inspirait, transformait la violence à son endroit en une puissance qui l'élevait au-dessus de tous : «- Pourquoi tu aimais qu'il t'effraie ? Je ne comprends pas. Tu aimais ça avoir peur ?» (2011, p. 58)

Battue, séquestrée et violée, Noé n'en était pas moins celle qui dominait tous les hommes qui l'asseyaient. Capable de jouir de tous les assauts, elle traverse indemne le récit, si vivante qu'elle peut se réincarner dans une publication ultérieure. Dans Les Sangs non plus, malgré les apparences, les femmes ne sont pas vulnérables.

Je jouis en continuant mes exercices, sans me toucher, rien qu'en pensant; je jouis de me trouver belle dans les yeux de l'homme imaginaire, et je jouis surtout de savoir que c'est dans la souffrance de tous mes membres que je suis vraiment désirable, quand les muscles sont tendus et le corps dessiné par l'effort. (Wilhelmy, 2013, p. 58)

Il serait faux de dire que les textes de Wilhelmy défendent ou réhabilitent ces attitudes de violence envers les femmes, mais ils les font exister : moins dans les domaines du narratif 
que dans ceux du sensoriel d'ailleurs, ce qui est singulier pour des récits que l'on associe au conte. Pourtant, Oss, Les Sangs et Le corps des bêtes sont peu narratifs, les histoires en tant que telles ne sont pas résolues, progressent peu. Les récits évoluent en volutes; à terme, en demeure des mouvements, une musicalité, plus que des types humains ou pire, une morale. Dans ces récits-croquis la narration est organique, on reçoit chaque épisode - les viols, incendies, noyades, morts - comme un coup, un vomissement, un mal de ventre, une égratignure. C'est que le style d'Audrée Wilhelmy a cette capacité de rendre l'horreur esthétique. Il y a, de fait, dans ses récits une musicalité qui permet d'accepter les pires événements. Le choix du vocabulaire, souvent vieilli, savant ou rare, détourne la narration de la violence évoquée. Le contexte privilégié, parce que les faits se déroulent dans un monde imaginaire, naturel et brut, favorise également cette réinterprétation des rapports de force.

C'est ici que la forme du conte lui offre une liberté tant spatiale que temporelle. Les univers sont les siens ; l'époque, une sorte de XIXe siècle anachronique. Les contraintes sont ainsi réduites à celles qu'elle se crée. Les attentes de la représentation ne sont ni québécoises ni françaises ; les cités sont imaginaires, affranchies de normes sociales et culturelles. Dans ses croquis que l'auteure aime partager sur son blogue, les villes sont complexes, sinueuses ; dans le texte, on y voyage plus simplement. Le décor est malléable : on reconnait la pierre, la plage, la terre, la fête foraine, et on tresse autour de ces lieux les souvenirs qui sont les nôtres - tant littéraires que réels - sans que le texte ne mette à mal ces associations.

Quand on l'interroge sur la place de son écriture aux côtés de celles d'autres jeunes auteurs québécois, elle répond :

Je pense que la génération d'auteurs qui émerge en ce moment en est une qui se décrit plus facilement par la négative. C'en est une qui s'est détachée de la question 
identitaire. Dans les années 60 et 70, l'identité québécoise occupait l'essentiel du discours littéraire. Les décennies post-référendaires ont pour leur part été témoin du questionnement identitaire des minorités (littérature migrante, féministe, homosexuelle, etc.). La littérature contemporaine, celle de cette nouvelle génération mentionnée dans ta question, est marquée par un délaissement de cette question d'identification à une petite ou une plus grande communauté. Je pense que notre génération n'a plus besoin de la fiction pour revendiquer le droit d'exister au sein de telle ou telle collectivité.

Il est par ailleurs difficile de définir de grandes lignes qui caractériseraient cette génération d'auteurs comme nous l'avons fait avec les précédentes. Aucun type d'écriture ne domine la scène littéraire; les romans publiés depuis l'émergence des nouvelles maisons d'édition (je pense notamment à Alto, au Quartanier et au Marchand de feuilles, mais il y en a bien d'autres) mettent en scène toute sorte d'univers très différents les uns des autres; la langue même, qui va du joual au français international, est entièrement libérée des contraintes qui la régissait il n'y a pas si longtemps encore.

C'est difficile, donc, de dire que je m'identifie à une génération qui est caractérisée par la possibilité de faire absolument tout ce qu'elle veut. Je pense que, d'une certaine manière, je suis assez loin de ce qui est populaire présentement, parce que je ne joue pas avec la langue orale, et que ce que j'écris ne s'inscrit pas du tout dans un Québec réel ou fictif.

Même chose pour la question de l'envie de me distinguer : comme je ne ressens pas le besoin de faire comme les autres, je n'ai pas non plus un besoin aigu de faire différemment des autres. J'ai l'impression qu'en faisant simplement ce qui me plait et ce qui me ressemble, je me creuse une place qui est la mienne, qui est celle qui me convient.

La fluidité de l'écriture d'Audrée Wilhelmy va de pair avec un accueil inconditionnel

de la nature. Il n'y a pas ici de sélection naturelle ; la vague est aussi écume ; le bois, fongus ;

l'animal, carcasse ; le suave, puanteur. Chaque élément a sa place, joue un rôle dans cet organisme. L'animalité des personnages est ainsi une forme d'harmonie, un respect de l'instinct qui leur permet souvent de se fondre dans leur habitat. Le retour à la nature est un accomplissement non pas une régression. En accepter la force, la dureté, l'ambition, est le propre des élus. Les personnages d'Audrée Wilhelmy sont tous des exceptions.

De fait, des conditions de la lecture, c'est la sauvagerie et la vivacité qu'on retient. Les contes traditionnels, on le sait, ont été édulcorés pour nos enfants jugés fragiles. Audrée 
Wilhelmy, en ce sens, revient à la source : la violence, la brutalité, la cruauté humaine sans fards et sans explications, les pulsions destructives également, motivent ses récits.

Ainsi par exemple dans Les Sangs, les femmes connaissent les désirs meurtriers de l'ogre mais n'en sont pas moins séduites. Amoureuses au point de vouloir surpasser toutes les épouses passées et futures, elles usent de génie pour que leur assassinat soit inoubliable, inégalé et elles en contrôlent tous les paramètres, l'ogre devenant par un pervers renversement un outil plus qu'un acteur dans leur mort. Les femmes de Féléor se sacrifient pour le plaisir de l'homme, sorte de métaphore excessive de la sexualité, mais le don est consentant.

La violence dans Les sangs est ainsi moins dans le meurtre que l'ogre perpétue sur les femmes de sa vie, que dans la compétition qu'elles se livrent. C'est pour surpasser les autres femmes qu'elles acceptent de lui donner leur vie. Être inoubliable, mais surtout effacer les autres. La lutte est intemporelle : « Mais après qu'il m’aura tuée, aucune femme, ni riche, ni pauvre, ni belle, ni parfaite, ne pourra se mesurer à moi. Même pas vous à qui j’écris. Je serai la seule à occuper ses rêves. Personne n'y arrivera comme moi ». (Wilhelmy, 2013, p. 65)

Si la diégèse est plus rebondissante dans Les Sangs que dans Oss ou dans Le corps des bêtes, il n'en demeure pas moins que la puissance des sept femmes de Barbe-bleue tient davantage à leur esthétisation qu'à leurs actions. Femme-liane, femme-sorcière, femmemachine, les épouses sont des allitérations, elles sont belles comme le grondement du tonnerre, spectaculaires comme le sang qui coule, ou la plaie qui s'infecte : « Je caressai des dents la plante du pied et pressai ma langue sur l'orteil estropié [...] Puis mes dents trouvèrent une cloque ronde, pleine, que je fis éclater entre mes canines ». (Wilhelmy, 2013, p. 72) 
Elles embrassent leurs inclinaisons même les plus funestes, et on sent que la démarche convient à l'auteure qui ne fait pas non plus de concessions, au nom d'un politiquement correct qui voudrait par exemple que triomphe une idéologie féministe univoque. Que faire de ces femmes soumises qui se laissent battre, violer, tuer? Ces femmes qui se taisent? Comment peut-on porter un regard féministe sur ces femmes violentées?

Sur la monstruosité complexe de ses personnages, Audrée Wilhelmy explique :

On pouvait autrefois passer des heures dans les files d'attente des cirques, pour observer ce qu'on appelait alors les « monstres de foire ». Si le concept en luimême soulève nombre de questions éthiques, il en dit également long sur le pouvoir d'attraction qu'exerce la « différence » sur l'Homme. Je pense que le dégoût éveille en chacun des pulsions très fortes, et suscite une fascination que le normal, le banal, le quotidien ou le beau ne peuvent pas déclencher. Dans l'optique de la monstruosité, je crois qu'il faut aborder les personnages de Les sangs de la même manière. Sans être séduisants - leur folie est trop sordide pour ça -, ils demeurent mystérieux parce que tellement différents de tout ce que nous sommes habitués de voir. Leurs actions surprennent et donnent parfois la nausée, mais le lecteur continue de tourner les pages malgré tout et se trouve captivé par l'intensité de leurs pulsions.

Ce qui faisait sensation, me semble-t-il, avec les monstres de foire, tenait au côté humain de cette monstruosité-là. La femme à barbe est une femme, comme celles qui payent pour la voir, mais elle incarne aussi autre chose : l'exception à la norme. La femme ordinaire peut se retrouver en elle, le monstre lui renvoie l'image de ce qu'elle aurait pu être. L'homme y devine les traits de sa conjointe, sa fille, sa mère, et c'est dans cette familiarité-là que naissent les plus vives réactions. Il n'y a rien de plus fort que de se reconnaître soi-même dans la monstruosité d'autrui. Dans Les Sangs, le mécanisme d'attraction fonctionne de la même manière. Les pulsions destructrices des personnages se révèlent d'une violence inouïe parce qu'elles ont été amplifiées par le prisme de la fiction, mais cela reste des pulsions que chacun porte en soi, à divers degrés.

Les personnages de Les Sangs ne sont donc ni séduisants ni repoussants : ils fascinent, parce qu'ils sont le miroir déformé de nos propres pulsions.

Ni prodiges, ni erreur de la nature, mais, comme je le disais plus tôt, simples reflets déformés - amplifiés - des pulsions qui habitent chacun. Malgré le côté extrêmement singulier et inquiétant de mes personnages et, plus largement, de mon œuvre, je voudrais, à plus long terme, qu'on ait l'impression d'une œuvre qui parle de l'Homme en posant un regard différent, mais sensible, sur toute la partie intérieure de la vie psychique. Les personnages de Les Sangs, ceux d'Oss et aussi ceux du roman à venir sont des figures de la transgression, mais l'humanité repose précisément sur la transgression - ou plutôt sur la conscience d'une possible transgression. Je veux 
que la fiction me serve à parler de l'Homme, sans pour autant écrire des textes qui s'inscrivent dans un cadre réaliste. Wilhelmy ajoute :

Ils ne sont pas objets au point où peuvent l'être les personnages d'un conte, ou par exemple ceux d'Oss, dans lequel une sorcière, un prêcheur, un clown remplissent précisément les rôles de sorcières, prêcheur et clown, et ne s'éloignent pas de leur fonction narrative. Par contre, les personnages de Les sangs demeurent unidimensionnels et, par extension, plus objet que sujet. Un seul attribut les définit et toute leur identité repose sur cette marque singulière : Mercredi est l'imagination; Constance, la toxicomanie; Abigaëlle, le perfectionnisme; Frida, la colère; Phélie, la curiosité; Lottä, le narcissisme; Marie, le travestissement. À l'extérieur de ces traits caractéristiques, les personnages sont peu de choses. J'ai voulu construire des êtres « plats » qui illustrent un enjeu qui les dépasse largement. Ils ont quelque chose des marionnettes d'un ventriloque, et ne sortent pas de la trame déterminée par la Grande Histoire qui les unit et qui a pour objectif d'explorer les pulsions humaines. L'invention de personnages trop complexes, dont le tempérament aurait revêtu plusieurs facettes, aurait diminué le rôle introspectif du texte, et aurait desservi le propos principal du roman.

Audrée Wilhelmy n’est pas Josée Yvon, pourtant, leurs femmes mutilées lèchent leurs plaies de concert. Audrée Wilhelmy n'est pas Anne Hébert, mais le pouvoir qu'elles reconnaissent à leurs femmes-sorcières est cohérent. Audrée Wilhelmy n’est pas MarieClaire Blais, mais la perversité des désirs féminins qu'elles accueillent se comprennent. Audrée Wilhelmy respecte les portes qu'ont ouvertes ces grandes féministes dans les années 1960. Elle ne les emprunte pas toujours, mais ses personnages rejoignent les autres grandes figures du féminisme québécois. Comme les femmes-kamikazes d'Yvon, Julie Labrosse des Enfants du sabbat ou Héloise d'Une saison dans la vie d'Emmanuel, Noé, Constance, Abigaëlle ou Mie font leur marque, deviennent les emblèmes d'une libération. Et à ce titre, Noé mène. La scène où elle dépèce une baleine peut servir de métaphore pour comprendre toute l'esthétique de Wihelmy :

Noé plante un grand crochet dans le flanc découpé, à l'endroit où, il n'y a pas cinq minutes, pendait mollement la queue. Elle monte sur la carcasse, puis, faisant contrepoids avec son corps, elle tire la peau qui se détache en un interminable bandeau rose. Quand elle arrive au bout, avec la lame elle sépare le cuir du gras ; c'est un tissu long de huit mètres et large de deux. Dans quelques jours, elle le frottera dans la mer 
pour enlever les résidus, elle le fixera à une base de bois, l'écharnera, elle fera fondre le cerveau de la baleine dans l'eau et tannera la peau, attendra une semaine encore, puis elle la battra, l'enfumera, enfin elle la clouera sur le sol de la cabane pour bloquer le froid, l'hiver; avec les restes, la Vieille fera des souliers, des besaces, des ceintures. (Wilhelmy, 2017, p. 96.)

Dominant le corps monstrueux, gigantesque, pour en faire une nourriture pour sa famille, Noé est femme. Indifférente au sang, et à la puissance du corps de la bête, cette femme affirme la force de son genre. Parce que c'est à des scènes de préparation de repas, à des scènes d'accouchement aussi que renvoie ce tableau. Mais voilà que mon fil se casse, que mon tissu se troue. Car en effet, quand il est question de prendre soin, l'œuvre de Wilhelmy prend un revers surprenant.

Car Noé néglige sa fille. Elle la laisse évoluer seule dans cette nature aride et dangereuse où le renard est homme et animal à la fois. Quand Mie lui demande conseil, quand elle tente désespérément d'obtenir l'attention de sa mère, Noé l'ignore, reste muette. L'enfant s'est élevée seule, comme sa mère l'a fait avant elle. Ces femmes fortes ne se plient pas aux exigences de nos sociétés patriarcales. Elles établissent leurs propres règles, leur propre code de conduite en dépit des attentes d'un social qui de toute façon est le nôtre et pas le leur.

Le care, ou « prendre-soin », est une éthique qui vise à valoriser les besoins plutôt que les droits, besoins d'ordre psychologique (besoins d'attachement, de respect et de sécurité) ainsi que d'ordre physiologique (chaleur, hygiène et nourriture), afin de mettre en relief le travail de ceux qui au quotidien répondent à ces besoins et garantissent ainsi le bon fonctionnement de toute société.

[L'éthique du care] repose sur ce constat : pour que certains parviennent à réaliser l'idéal occidental de l'individu libre, entrepreneur et volontaire, sue lequel repose l'édifice idéologique et économique néolibéral, il faut que d'autres, dans l'ombre, garantissent l'entretien des conditions matérielles de cette réussite. Par le soin aux enfants, aux malades, aux personnes âgées, le souci de l'alimentation, de la santé et de 
l'hygiène, l'entretien des lieux de vie et de travail, ils libèrent et autorisent l'efficacité de ceux qui produisent les richesses. (Snauwaert, 2015, p. 21)

Cette éthique reconnaît le caractère essentiel des activités de soin pour la préservation et la croissance du monde humain. La revalorisation participe de la lutte féministe et prolonge un combat qui concerne la reconnaissance d'un effort social souvent proprement féminin, sinon relevant de minorités visibles. Ce que Lori Saint-Martin (1992) nomme le métaféminisme, mouvement accueillant et englobant les luttes tant féminines que minoritaires ou queer, a déjà permis depuis plus d'une quinzaine d'années de repenser la hiérarchie sociale des tâches. Il ne s'agit donc pas d'entendre l'éthique du care comme une voix isolée mais comme le pan d'un discours féministe plus global.

Audrée Wilhelmy ne supporte pas les attentes concernant le rôle social de la femme que reconduisent les contes traditionnels devenus des classiques. Oss, Les sangs et Le corps des bêtes s'inspirent des codes du conte pour mieux marquer le moment où ils sont fracassés, développent les racines d'un mouvement de révolte à l'égard de certains motifs du conte.

Sorte d'exagération de l'éthique du mariage, Les sangs poussent à l'extrême le don de soi qu'implique le contrat. C'est le seul récit de Wilhelmy où il est question de mariage au sens où on l'entend. Oss et Le corps des bêtes se déroulent dans des circonstances trop éloignées du monde tel qu'on le connaît pour qu'on reconnaisse ce type traditionnel d'union. Le mariage est tordu dans Les Sangs selon les attentes du sado-masochisme. Féléor, le Barbebleue de Wilhelmy, est un homme riche et séduisant qui attire les femmes les plus belles de sa région. Celles-ci viennent à lui, s'offrent tout naturellement. De fait, dans le roman de Wilhelmy, les assassinats sont une initiative des femmes qui arrivent toutes, à l'exception de Marie des Cendres la dernière, à la conclusion que lui donner le plaisir de prendre leur vie 
constitue l'ultime jouissance qui leur permettra de devenir inoubliables et surtout de surpasser les femmes précédentes, expression ultime, voire caricaturale, du potentiel agentif.

Dans le contexte de ce roman, les épouses n'ont pas comme tâche de prendre soin de la maison. L'ogre a des domestiques, dont l'une, Marie des Cendres, sera sa dernière amante. Les épouses de Féléor ne lui feront pas non plus d'enfants, la consommation du mariage est vaine et purement sexuelle. Une seule, la veuve Frida, a eu trois enfants d'un premier mariage, mais ceux-ci ont grandi et elle n'a plus à prendre soin d'eux. D'ailleurs, elle garde rancune aux grossesses qui ont abîmé son corps :

J'ai agi en automate pendant quinze ans, réglée comme une horloge, toute entière dévouée aux besoins d'une maisonnée que je détestais pourtant. [...] J'ai eu toutes les responsabilités du mariage sans en avoir un seul avantage. Quand je me goinfre par terre comme une truie, entourée de ces poules parfumées qui me rappellent continuellement ma déchéance, il monte en moi une telle rage d'avoir été femme et jeune, mais d'avoir été engoncée dans le corps mou des grossesses et les voiles noirs du deuil ! Je ne pardonnerai jamais à mon mari ni à mes enfants la carcasse horrible héritée de ces années-là. (Wilhelmy, 2013, p. 80)

Tout à l'opposé, Abigaëlle est une ballerine acharnée qui travaille son corps sans relâche poursuivant des désirs de perfection qui séduisent l'ogre. Le travail qu'elle s'impose n'implique pourtant aucune responsabilité. Sa passion pour la danse est une discipline intense, non pas une corvée obligatoire ou utile. Les femmes de Féléor ont en effet en commun d'être totalement oisives, la seule exigence de l'ogre étant qu'elles écrivent un journal. Elles n'ont pas à prendre soin de la maison, ni des repas, ni des enfants, ni même de leur mari qui n'a que des besoins très limités sinon sexuellement, mais encore là, les relations sont souvent l'initiative des femmes. Féléor exige peu. Mais prend tout.

Dans tous les cas, prendre soin ne faisait pas partie de l'éthique de ces mariages. Au mieux, pourrait-on penser que la jouissance personnelle constitue l'unique promesse de ces 
unions. Car en effet, même si ces femmes donnent leur vie, se laissent assassiner pour le bon plaisir de l'ogre, c'est avant tout pour tenter de gagner la lutte qu'elles entretiennent avec leurs rivales (passées et futures). C'est une compétition que se livrent les femmes : c'est à qui sera la plus désirable dans la mort. Le sacrifice, atteignant ici une limite absolue, ne répond donc à aucune éthique, celle qu'a décrite Jacques Derrida entre autres. Pour reprendre les termes de la philosophie derridienne, le don que met en scène Wilhelmy dans son roman est institutionnalisé, programmé, ritualisé, il devient donc sacrifice, le concept, Derrida l'a bien montré, étant essentiellement lié à une reconnaissance, contrairement au don qui ne devrait souffrir aucune circularité : « Pour qu'il y ait don, il faut qu'il n'y ait pas réciprocité » (Derrida, 1993, p. 24). Si les femmes donnent leur vie à Féléor ce n'est pas par pure dévotion, mais bien parce qu'elles y gagnent quelque chose, en l'occurrence leur supériorité par rapport aux autres femmes. Il y a ici réciprocité puisque le sacrifice rapporte : elles se donnent mais gagnent ainsi une valeur dans la hiérarchie de l'ogre ; ces femmes ne sont ni généreuses ni dévouées, elles poursuivent une quête égoïste et orgueilleuse. A terme, c'est leur propre jouissance qui est en jeu.

Audrée Wilhelmy explique ainsi ce lien de son écriture avec les théories du care :

Cette qualité de la femme toute tournée vers les soins à prodiguer à son entourage (progéniture, mari ou amant) m'a toujours vivement dérangée, comme s'il était impossible, pour être une femme accomplie, d'exister à l'extérieur des autres. Les femmes de Les sangs - comme celles de mon prochain roman d'ailleurs transcendent cet enjeu : elles ne se positionnent pas contre le care, elles n'en ont tout simplement rien à faire. Leurs actions ne sont pas dictées par un engagement quelconque, mais par une liberté totale et une inconscience des codes de notre société (elles évoluent dans un monde distinct du nôtre, ce qui me permettait de contourner plus facilement ces enjeux).

Plus précisément, je dirais que les femmes de Les sangs sont éminemment égocentriques et s'intéressent bien davantage à elle-même qu'à autrui. Leurs pulsions et leurs désirs sont tournés vers l'intérieur, elles ne s'ouvrent à l'autre qu'à travers l'écriture. La perception qu'elles ont de leur mari est d'ailleurs souvent faussée par le 
regard qu'elles aimeraient que lui jette sur elles (il est souvent réduit au rôle de miroir). Certaines, comme Frida-Oum Malinovski, ne prennent même pas soin de leur propre personne et seraient bien en peine d'accorder du temps à autrui. Il y a bien des exceptions : Marie-des-Cendres, la dernière femme, contredit un peu ce modèle de femmes entièrement libres et introversives, mais cela reste principalement dû à sa fonction de femme de chambre : elle est naturellement amenée à prendre soin de son maître.

L'éthique à laquelle participe Wilhelmy n'est donc pas dans la lutte, mais dans la représentation. A tout le moins y a-t-il chez Wilhelmy une remise en cause des idées reçues sur la domination. Choisissant explicitement un conte où s'exprime originalement la suprématie masculine, par la figure d'autorité de Barbe-bleue, Wilhelmy montre l'envers du décor où cette suprématie n'existe que parce que des femmes y ont consenti, y trouvant leur compte dans une certaine mesure. Si les femmes d'Audrée Wilhelmy abandonnent leurs devoirs, c'est pour mieux reprendre leurs droits. Selon la même logique, les femmes sauvages de Oss ou du Corps des bêtes limitent leurs responsabilités au minimum : se nourrir, se vêtir. Le primitivisme de ces femmes, il faut le comprendre, est un choix, car si elles vivent dans des sociétés imaginaires décalées par rapport aux nôtres, il n'en demeure pas moins que les personnages secondaires qui évoluent autour de ces femmes respectent des codes que nous comprenons et auxquels seules les femmes ne se plient pas. Les hommes des récits parlent, vivent dans des maisons, marchent sur des routes, vont au travail, achètent des biens, ont des familles.

Les femmes fortes, les sorcières, des contes d'Audrée Wilhelmy n'ont pas les ambitions de Cendrillon. Les excès de leur éthique leur seront fatals, mais elles n'en sortiront pas moins gagnantes. Dans ces récits, les femmes fuient leurs responsabilités pour que triomphe leur propre plaisir. On comprend que la représentation de la fuite n'est pas du tout la même que dans un roman comme La femme qui fuit d'Anaïs Barbeau-Lavalette, par 
exemple, profondément ancré dans le politique et l'actualité québécoise, dans lequel l'abandon de la mère en quête de liberté personnelle traumatise la famille sur plusieurs générations. Les récits de Wilhelmy évoluent dans un univers parallèle, dont participe aussi le conte, dans lequel le temps et l'espace n'ont pas la même prise, ce que Marie-Louise van Frantz nomme « le nulle-part de l'inconscient collectif» (Jean, 1981, p. 361). La veuve Frida, emblématique, a détesté ses responsabilités de mère, d'épouse, de femme au foyer. Désormais, elle ne fera plus que manger et baiser, jusqu'à en mourir. Il n'y a pas dans cette construction narrative de trame sourde - les pauvres enfants de Frida, ou ceux de Noé, souffriront-ils de la déchéance / de la négligence de leur mère ? cette question n'a pas sa pertinence ici - toute la dynamique étant concentrée sur l'immédiateté de la narration, comme dans le conte. La crédibilité du récit n'est pas mise à l'épreuve de l'actualité, de sorte que la violence peut être transitive ; et c'est l'héritage du conte qui lui donne cette force, cette possibilité, notamment en ce qui a trait à la représentation du politique et aux cohérences du social qui n'ont plus de prises sur le récit.

\section{Conclusion}

Le destin des personnages influence ici la structure narrative. Dans le conte traditionnel, la circularité est liée à l'aspect oral du récit, qui pour être raconté plus facilement repose sur une structure cohérente et aisée à reproduire, voire à dériver. Chaque conteur s'approprie en effet le récit aux grés de ses envies, de ses trous de mémoires et de son public. On adapte, on oublie, on reprend, sans que l'essentiel de la structure ne soit nécessairement affecté, précisément parce qu'elle est faite pour être malléable. De fait, on peut considérer les contes comme des squelettes, des structures de créativité, plus ou moins élaborées qui invitent à la reproduction. C'est également sur cette garantie que se fonde la moralité des contes : ce qui 
est arrivé arrivera de nouveau. Les romans d'Audrée Wilhelmy reconnaissent la force de la circularité : les cahiers de chacune des femmes de Féléor reprennent les motifs du précédent, Le corps des bêtes poursuit un ouvrage initié dans Oss. L'auteure ne pervertit pas les contes traditionnels. Elle dispose d'une forme. Pas de folklore dans ces textes allitérés, mais des formules rituelles, rythmées. L'écriture est récital.

Cette structure narrative circulaire implique une grande violence, puisque dans le dénouement prévisible se cristallise des destins sans espoirs.

Les héroïnes refusent les conventions sociales établies par le régime patriarcal, et véhiculées par le conte traditionnel, mais puisqu'il s'agit essentiellement d'une réaction, elles semblent demeurer subordonnées à ce qu'elles rejettent. Les récits d'Audrée Wilhelmy brisent la dynamique et rejettent une moralité cristallisée par les contes traditionnels, voire refusent les lignes directrices des féministes, mais ne proposent pas une éthique de remplacement. Ce que fait Wilhelmy dans ses fictions est d'explorer le moment où des femmes décident de faire table rase, soit en niant ces besoins, soit en refusant d'y céder, au risque, bien sûr, de courir à leur propre perte. Comment alors faut-il interpréter cette multiplication de personnages féminins violentés, ces femmes fortes mais muettes ? Quelle éthique sociale est défendue dans ces textes?

Il semble que la réponse à cette question délicate se trouve dans la parole qu'ouvrent les récits. De l'oralité du conte traditionnel reste un lyrisme. Le style de Wilhelmy aboutit dans Le corps des bêtes à une forme d'incantation claquante. La répétition se trouvait déjà dans les textes précédents, mais ce dernier texte assume davantage cette force de la formule. C'est pourtant celui où les personnages parlent le moins. Partout, la parole est déficiente. La voix du texte n'en est pas moins forte. Les silences y sont retentissants, dérangeants, ils sont 
résistance et impertinence. La parole y est grognement, chanson, cri, râle. Le langage du corps rendu à l'esthétique : «En deux semaines, elle n'a pas dit un mot mais elle a chanté souvent, des chants dans une langue pleine d'accents secs ». (Le corps des bêtes, 2017, p. 19)

Ses fictions charrient des femmes : marrées rudes qui n’en épargnent aucune. Noé, Mie, Abigaëlle, Constance, Frida. On en retient la beauté, la bestialité, la force. Des sorcières, des vraies. Des femmes brutes et intelligentes, gardiennes d'un savoir qu'elles ne souhaitent pas toujours transmettre. Des femmes qui chantent, qui grondent, qui écrivent, qui s'accouplent, mais qui parlent peu. Des femmes qui dépècent des baleines. Des corps sales, des fluides, des pulsions qui résument chacune de nous et nous confondent. Elles ont des griffes, et des blessures qu'elles lèchent. On les attache, on les frappe, on les torture, on les noie, mais elles ne disparaissent pas. Leurs silences continuent de vibrer, pulser, grogner. Quand le livre se referme, il reste une danse, souple et sauvage : la violence brute du féminin.

\section{Bibliographie}

DERRIDA, Jacques (1993), Donner le temps. 1- La fausse monnaie, Paris, Galilée.

JEAN, Georges (1981), Le pouvoir des contes, Paris, Casterman, « E3: enfance-éducationenseignement $»$.

SAINT-MARTIN, Lori (1992), « Le métaféminisme et la nouvelle prose féminine au Québec », Voix et Images, vol. 18, $\mathrm{n}^{\circ}$ 1, (52). 
SNAUWAERT, Maïté (2015), « Une nouvelle éthique féministe. Les vertus de l'éthique du care contre les dérives de l'individualisme », La moitié du monde. Comment le féminisme pense la société, Revue Liberté, n 307, Printemps.

WiLHELMY Audrée (2011), Oss, Montréal, Leméac.

WiLhelmy Audrée (2013), Les Sangs, Montréal, Leméac.

WiLhelmy Audrée (2017), Le corps des bêtes, Montréal, Leméac.

\section{Notice biobibliographique}

Marie-Hélène Larochelle est professeure agrégée à l'Université York. Ses recherches portent sur la violence dans la littérature contemporaine. Elle est l'auteure des essais L'abécédaire des monstres. Fragments de Réjean Ducharme (PUL, 2011) et Poétique de l'invective romanesque, L'invectif chez Louis-Ferdinand Céline et Réjean Ducharme (YYZ, 2008). Elle a dirigé plusieurs dossiers de publication dont Le Dire-monstre (Tangence, 2009), Identités monstrueuses : violences et invectives dans le roman francophone européen (Présence francophone, 2010) et Monstres et monstrueux littéraires (PUL, 2008). Elle est également l'auteure d'un roman, Daniil et Vanya (Québec Amérique, coll. "Littérature d'Amérique », 2017), et de deux nouvelles, Crudité (dans Monstres et fantômes, Québec Amérique, coll. « La Shop », 2018), et Phenix (dans Stalkeuses, Québec Amérique, coll. " La Shop », 2019) qui travaillent la mise en esthétique de la violence. 